\title{
One Kairomone and Multiple Effects in Daphnia Species - 5 $\alpha$-Cyprinol Sulfate Induces Morphological Defenses in the Invasive Species Daphnia lumholtzi
}

\section{OPEN ACCESS}

Edited by: Sebastian Kruppert, University of Washington, United States

Reviewed by: Blake Matthews,

Swiss Federal Institute of Aquatic Science and Technology, Switzerland Taisen Iguchi,

Graduate University for Advanced Studies (Sokendai), Japan

*Correspondence: Meike Hahn meike.hahn@uni-koeln.de

Specialty section: This article was submitted to Behavioral and Evolutionary Ecology, a section of the journal Frontiers in Ecology and Evolution

Received: 29 October 2021

Accepted: 10 February 2022

Published: 03 March 2022

Citation:

Hahn M and von Elert E (2022) One Kairomone and Multiple Effects in Daphnia Species - $5 \alpha$-Cyprinol Sulfate Induces Morphological Defenses in the Invasive Species

Daphnia lumholtzi.

Front. Ecol. Evol. 10:804521. doi: 10.3389/fevo.2022.804521

\author{
Meike Hahn* and Eric von Elert \\ Aquatic Chemical Ecology, Department of Biology, University of Cologne, Cologne, Germany
}

Recently, the first chemical cues, which induce defenses in freshwater zooplankton of the genus Daphnia have been identified. Still it is unknown how general these socalled kairomones are and if they are perceived by and thereby benefitting invasive species. We here report the chemical identity of the fish-derived chemical signal that induces morphological defensive structures in the invasive species Daphnia lumholtzi. We used bioassay-guided isolation (LC-MS) of incubation water of fish and identified the bile salt $5 \alpha$-cyprinol sulfate ( $5 \alpha$-CPS) as the kairomone. We confirmed this finding by bioassays, in which $D$. lumholtzi was exposed to purified $5 \alpha$-CPS sulfate from fish bile and showed that $10 \mathrm{pM} 5 \alpha$-CPS induces significantly elongated helmets and spines in relation to body length. These results identify on one hand another Daphnia species besides Daphnia magna, which is responding to this particular fish-borne kairomone, and on the other hand they reveal another defense that the kairomone induces besides diel vertical migration (DVM). Taken together a high evolutionary conservancy of the molecular mechanism behind inducible defenses in Daphnia spp. against fish can be deduced. It is reasonable to expect that $5 \alpha$-CPS is involved in further predator-prey communication between Daphnia and fish, which may be of special ecological relevance with regard to invasive species as $D$. lumholtzi.

Keywords: invasion, invasive species, chemical communication, infochemical, plasticity, Daphnia, inducible defense

\section{INTRODUCTION}

Freshwater crustaceans of the keystone genus Daphnia are known for a variety of inducible defenses which protect them from predation and which are induced by predator derived infochemicals (Tollrian, 1994; Von Elert and Loose, 1996; Effertz and Von Elert, 2014). Though the first of chemical cues, which induce particular defenses in Daphnia, have recently been identified (Weiss et al., 2018; Hahn et al., 2019), it is still uncertain how universal these so-called kairomones are and if they are perceived by and thereby benefitting invasive species like Daphnia lumholtzi as well.

While Chaoborus sp. was shown to exude glutamine-conjugated fatty acids, which induce neck teeth formation in $D$. pulex (Weiss et al., 2018), cyprinid fish were shown to exude $5 \alpha$-cyprinol sulfate ( $5 \alpha$-CPS), which induces the predator-avoidance behavior "diel vertical migration" (DVM) in Daphnia magna (Hahn et al., 2019). The fact that different kairomones are utilized by Daphnia 
to chemically perceive the presence of an invertebrate or a vertebrate predator is not surprising considering that Daphnia sp. respond with different defenses to them. These different defenses are needed due to differing foraging modes of Chaoborus larvae and fish (Brett, 1992). In accordance with this, it has already been shown that independent internal signaling pathways are involved in the response to chemical cues from Chaoborus and fish in Daphnia (Weiss et al., 2012).

The universality of the DVM-inducing kairomone $5 \alpha$-CPS for Daphnia has not yet been investigated, as it remains to be tested (i) if $5 \alpha$-CPS is inducing not only DVM but as well other defenses against fish, and (ii) if $5 \alpha$-CPS is as well active in Daphnia species other than D. magna.

In response to fish kairomones Daphnia may respond with different inducible defenses, i.e., the induction of DVM, of lifehistory changes (LHCs) or of morphological changes (Dodson, 1988; Macháček, 1991; Tollrian, 1994). The finding that these different defenses do not strictly co-occur across different Daphnia genotypes, i.e., that a decoupling of these defenses is observed (Boersma et al., 1998), makes it conceivable that these different defenses are controlled by different mechanisms. Investigations of the underlying neurophysiological mechanisms of perception of fish kairomones do not necessarily point at different neuronal signaling pathways involved in fish-mediated induction of DVM and LHCs: Weiss et al. (2012) revealed that GABAergic signaling was involved in fish-mediated lifehistory changes, and in a comparable approach for fish-mediated induction of DVM (Bedrossiantz et al., 2021) reported the involvement of GABAergic and muscarinic cholinergic signaling. Hence GABAergic signaling seems to be involved in induction of both DVM and LHCs, and one straightforward explanation for the above-mentioned decoupling of these defenses across Daphnia genotypes is that induction of DVM and LHCs rely on different kairomones released from fish. This reasoning is supported by Von Elert and Stibor (2006) who used a D. magna clone that responds to fish-borne kairomones with induction of DVM and LHCs and preliminarily characterized chemical features of the LHC inducing fish kairomone. After fractionation by HPLC, DVM-inducing and LHC-inducing activity were confined to different fractions, which indicated that different kairomones were causing DVM and LHCs.

It has been shown that fish-borne chemical cues induce different defenses in different Daphnia species like for instance in D. magna and D. lumholtzi (Tollrian, 1994; Von Elert and Loose, 1996). While the induction of DVM in D. magna has been attributed to the fish-borne kairomone CPS (Hahn et al., 2019), it is still not known, which fish-borne compound induces the protective helmets and tail spines in the invasive species D. lumholtzi. By answering this question, one can identify a compound that benefits an invasive species and furthermore gain insights into how universal $5 \alpha$-CPS as a fish-borne kairomones is in the genus Daphnia. Furthermore, these investigations can indicate, if a signaling pathway different from that involved in fish-mediated LHC and DVM induction, is underlying morphological defenses against fish in Daphnia.

The inducible elongation of the helmet and tail spine of D. lumholtzi has been shown to protect them from predation by fish and Chaoborus larvae (Engel et al., 2014). Under continued exposure to fish kairomones, this induction of morphological defenses in D. lumholtzi is transferred to the next generation resulting in an earlier ontogenetic onset and enhanced magnitude (Graeve et al., 2021). Furthermore, fish-induced lateral deformations at the fornices (appendages of the head capsule) were observed in D. lumholtzi, resulting in a significantly increased lateral width of the animals (Horstmann et al., 2021). D. lumholtzi has invaded large parts of the United States over more than 25 years (Sorensen and Sterner, 1992; Beyer and Hambright, 2019). This invasion success of $D$. lumholtzi has been attributed to an elevated temperature tolerance of $D$. lumholtzi and to the inducible formation of defensive spines in presence of predatory fish (Engel and Tollrian, 2009, 2012; Engel et al., 2014). This potential importance of inducible morphological defenses for the invasion success of D. lumholtzi makes it particularly intriguing to identify the responsible kairomone.

In order to gain knowledge about the generality of fish kairomones that induce different defenses in different Daphnia species, we here identify the fish kairomone to which the invasive species $D$. lumholtzi responds. Even though, $5 \alpha$-CPS has already been identified as a fish-borne kairomone used by Daphnia, we do not simply test the effect of $5 \alpha$-CPS on $D$. lumholtzi, but identify the one active compound from extracted fish incubation water, which is present in sufficiently high concentrations to explain the induction of morphological defenses in D. lumholtzi. We initiate kairomone identification by investigating the effect of extracted fish incubation water and HPLC fractions thereof on helmet and tail spine length of D. lumholtzi. The extract, the respective HPLC fractions and the purified $5 \alpha$-CPS had already been investigated in the context of DVM induction in D. magna (Hahn et al., 2018, 2019).

\section{MATERIALS AND METHODS}

\section{Cultures}

Up to 15 synchronized Daphnia lumholtzi of a clone from Joe Pool Lake, Texas $\left(32.35^{\circ} \mathrm{N}\right)$ (Whittington and Walsh, 2015) were kept in $300 \mathrm{~mL}$ aerated, membrane-filtered $(0.45 \mu \mathrm{m})$ tap water and fed $2 \mathrm{mg}$ carbon $\mathrm{L}^{-1}$ of Cryptomonas sp. every other day.

Cryptomonas sp. (strain SAG26.80 Culture Collection of Algae Goettingen, Germany) was cultured semi-continuously in sterile Cyano medium (Von Elert and Jüttner, 1997) with vitamins according to Hahn and von Elert (2020).

\section{Bioassay}

D. lumholtzi originating from the 3rd clutch of synchronized mothers within the last $24 \mathrm{~h}$ were exposed to the respective treatments in groups of two in $250 \mathrm{~mL}$ water, fed $2 \mathrm{mg}$ carbon $\mathrm{L}^{-1}$ of Cryptomonas sp. daily and transferred to fresh treatments every other day. When individuals carried the first clutch, digital images were taken (magnification of 20X) using a dissecting microscope, and the length of helmet, body, tail spine and clutch sizes were measured using ImageJ (1.51 $\mathrm{k}$, Bethesda, Maryland, United States). For determination of helmet and body length (see Figure 1B). The juvenile somatic 

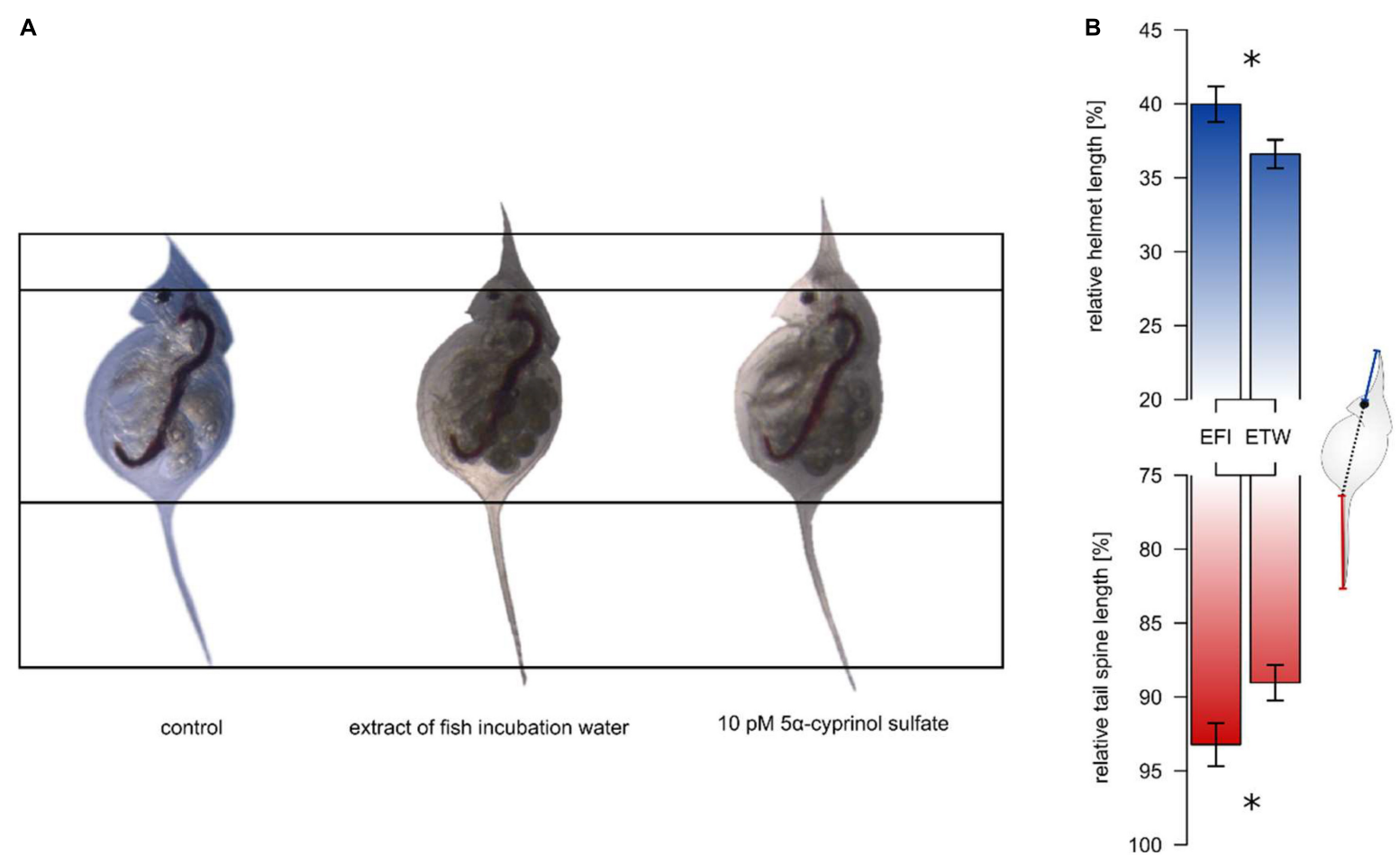

FIGURE 1 | Effect of extract of fish incubation water on helmet and tail spine of Daphnia lumholtzi. The animals were exposed to $\mathrm{C}_{18}$-solid phase extract of either incubation water of roach (Rutilus rutilus, EFI) or of tap water, aged for $24 \mathrm{~h}$ (ETW, control). EFI leads to relative elongation of helmets and tail spines (A). Barplots in (B) show the relative length (length/body length ${ }^{*} 100$ ) of helmet (blue) and tail spine (red) as mean \pm SD derived from animals at time of their first reproduction (B). Asterisks indicate significant differences after $t$-test $(p<0.05, n=3)$. The Daphnia scheme in (B) depicts the measurement of absolute helmet (blue) and tail spine (red) lengths, which were used for the calculation of relative lengths.

growth rate "g" of animals was calculated based on dry weight according to Wacker and Elert (2001).

\section{Statistics}

Helmet and tail spine length of individuals at their first reproduction were divided by the body length and subsequently multiplied by 100 to obtain relative helmet and relative tail spine length (Engel and Tollrian, 2009). Those and data on somatic growth rate were averaged for all animals from one experimental jar. Potential effects of the investigated fish exudates on relative length of helmets and tail spines, body length and somatic growth rate were investigated by ANOVA (after confirmation of homoscedasticity of the data by Levene's test) or $t$-test using the software $\mathrm{R}$ version 3.6.2 (R Core Team, 2019) and the packages agricolae version 1.3-2 and car version 3.0-6.

\section{Extraction of Fish Incubation Water and HPLC Fractionation}

An extract of fish incubation water (EFI) of Rutilus rutilus was obtained using a $\mathrm{C}_{18}$ solid phase according to Hahn et al. (2019). EFI was separated into six fractions by UHPLC (Accela 1250, Thermo Fisher Scientific, Waltham, Massachusetts, United States) according to Hahn et al. (2019), and analytes within the mass range $m / z \quad 120-1,500$ were measured in the negative electrospray ionization (ESI) mode and detected with one scan per second. Ion source parameters were: Sheath gas flow rate: 35, aux gas flow rate: 5 arbitrary units of the device, spray voltage: $4.3 \mathrm{kV}$, capillary voltage: $-60 \mathrm{~V}$, tube lens voltage: $200 \mathrm{~V}$, skimmer voltage: $-20 \mathrm{~V}$, capillary temperature: $325^{\circ} \mathrm{C}$. Mass accuracy was calibrated daily using the Pierce LTQ ESI negative calibration mixture.

\section{Database Research}

In this study the same HPLC-fraction (fraction 3) of EFI as in Hahn et al. (2019) proved to be active; hence identification of the most likely candidates of the kairomone was performed as detailed there. The software MassWorks (Cerno Bioscience, Las Vegas, Nevada, United States) was used to extrapolate from measured exact $m / z$ ratios and isotope patterns to sum formulas. For these sum formulas a database search was performed using the online database ChemSpider. Bile salts present in EFI have already been identified in Hahn et al. (2019) by LC-MS.

\section{Quantification of Bile Salts}

Bile salt concentrations in EFI and carp bile extract have already been quantified in Hahn et al. (2019). The bile salt taurochenodeoxycholic acid (TCDCA) was quantified by the standard addition method, and $5 \alpha$-CPS was quantified as equivalents of cholesteryl sulfate (Hahn et al., 2019). Prior to LC-MS analysis, glycocholic acid hydrate (GCA, > 95\% purity, 


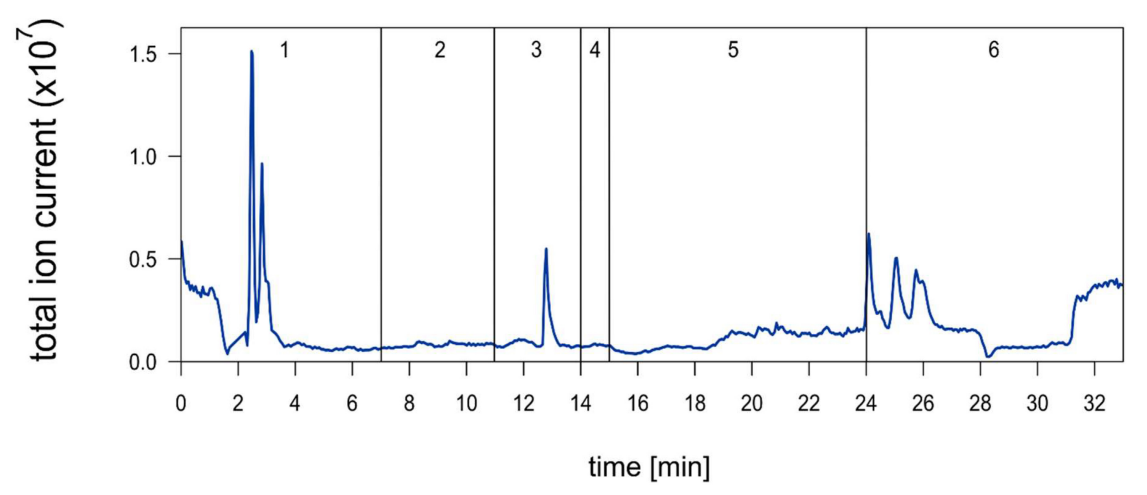

FIGURE 2 | Chromatogram of extract of fish incubation water (EFI) (Hahn et al., 2019) published under the Creative Commons Attribution 4.0 International Public License (CC-BY 4.0), (https://creativecommons.org/licenses/by/4.0/), no changes were made. Negative total ion chromatogram derived from ESI-MS of EFI after separation on a $250 \mathrm{~mm} \times 4 \mathrm{~mm}$ reversed phase column. Vertical lines and numbering indicate collection of fractions 1-6.

Sigma Aldrich, St. Louis, Missouri, United States) was added to the samples to account for variability of the mass spectrometer's sensitivity. This variability bias was eliminated by normalization of the analytes' peak areas to the respective GCA peak area of a sample. Calibration curves for quantification of analytes in EFI were measured in the background of EFI in triplicates to compensate for effects of its matrix on the device's sensitivity.

\section{Extraction of Fish Bile (Cyprinus carpio) and Purification of $5 \alpha$-Cyprinol Sulfate}

Briefly, bile of Cyprinus caprio (Cyprinidae) was obtained from the gall bladder and extracted according to Hahn et al. (2018). NMR analysis confirmed the identity of $5 \alpha$-CPS, and LC-MS demonstrated its purity. The same solvent gradient as described in Hahn et al. (2019) was applied, but a $12.5 \times 2 \mathrm{~mm}$ reversed phase (Nucleosil $\mathrm{C}_{18}, 100-3$, Macherey-Nagel, Düren, Germany) was used.

\section{RESULTS}

We showed that fish-derived kairomone(s) that induce morphological defenses in Daphnia lumholtzi can be extracted by $\mathrm{C}_{18}$ solid-phase from incubation water of roach (Rutilus rutilus, Cyprinidae): The exposure of D. lumholtzi to an extract of fish incubation water (EFI) resulted in relative elongation of helmet and tail spine compared to those of animals exposed to methanolic extract of tap water (ETW) (Figure 1). EFI caused an elongation of the relative helmet length from 36 to $40 \%$ ( $t$-test, $\left.t_{4}=-3.79, p<0.05\right)$ and of the relative tail spine length from 89 to $93 \%$ ( $t$-test, $\left.t_{4}=-3.83, p<0.05\right)$, and elongated the absolute body length (Supplementary Table 1). Using the methanolic control extract ETW as negative control, neither methanol, which served as matrix for both extracts, nor compounds in the fish incubation water that did not interact with the solid phase (flow through), affected any morphological parameters (Supplementary Figure 1 and Supplementary Table 2). The absence of activity in the flow through demonstrates that all morphogenetic activity has been extracted by the $\mathrm{C}_{18}$ solid-phase and is therefore entirely contained in EFI. Since methanol and ETW did not differ in their effect on morphology of D. lumholtzi, methanol was from then on used as negative control in the subsequent bioassays.

EFI was chromatographically separated into six fractions by Hahn et al. (2019; Figure 2) and a 1:5 dilution thereof was prepared. Out of these six fractions only fraction 3 induced an increase of the relative helmet and tail spine length of $D$. lumholtzi to the same extent as crude 1:5 diluted EFI (in this experiment called EFI) and to significantly higher values than the control (Table 1). The relative helmet length of D. lumholtzi exposed to fraction $3(\sim 48 \%)$ was comparable to that of animals exposed to crude EFI (52\%) and 12\% longer than that of animals exposed to the negative control $(\sim 36 \%)$. The same pattern was observed for the relative tail spine length: Fraction 3 was the only fraction which induced a relative tail spine length ( $98 \%)$ not-distinguishable from that in responses to EFI $(\sim 100 \%)$ and significantly longer than in the negative control $(\sim 85 \%)$ (Table 1). Absolute body length did not differ between animals.

Prior examinations of EFI from roach and bile extract of carp by ESI-LC-MS analysis and NMR have already shown that the most abundant compound present in fraction 3 is the bile salt $5 \alpha$-cyprinol sulfate ( $5 \alpha$-CPS) (Hahn et al., 2018, 2019). This finding drew attention to the group of bile metabolites in general and revealed the presence of another bile salt, TCDCA, in fraction 3 (Hahn et al., 2019).

Quantification of $5 \alpha$-CPS in carp bile extract and EFI allowed us to test for the effect of both extracts on the morphology of $D$. lumholtzi, when applied in volumes corresponding to comparable $5 \alpha$-CPS concentrations. The bile extract induced a significant increase of the relative helmet and tail spine length $(14,9 \%)$, while body length was not affected (Supplementary Figure 2 and Supplementary Table 3). These effects on relative helmet and spine length were similar to those obtained with EFI (Supplementary Figure 2), which supports a causality between the elongation of helmets and spines and the two bile salts in fraction 3. Previous quantification of $5 \alpha$-CPS in carp bile and EFI (Hahn et al., 2019; Table 2) revealed that in bioassays with EFI and with a deliberately chosen dilution of bile, comparable 
$5 \alpha$-CPS concentrations were present (bile: $1.13 \pm 0.02 \mathrm{nM}, \mathrm{EFI}$ : $1.03 \pm 0.08 \mathrm{nM}$ ), which further supported that elongation of helmets and spines may be caused by the two bile salts in fraction 3 , i.e., $5 \alpha$-CPS and TCDCA.

Subsequently, dose-response experiments were performed with both compounds of interest, $5 \alpha$-CPS and TCDCA. Neither $5 \alpha$-CPS nor TCDCA influenced the body length of animals (Supplementary Tables 4,5 ). In case of $5 \alpha$-CPS, relative helmet length of $D$. lumholtzi exposed to a $5 \alpha$-CPS concentration $\geq 10$ pM could not be distinguished from that of animals exposed to EFI, corresponding to a $5 \alpha$-CPS concentration of $1.03 \pm 0.08$ $\mathrm{nM}$ (Figure 3A). The threshold concentration of $5 \alpha$-CPS for elongated relative helmet length was thus 100 times lower than the $5 \alpha$-CPS concentration in EFI. Relative tail spine length was as well equally elongated as in the presence of EFI if 10 pM $5 \alpha$-CPS were applied (Figure 3A). Exposure of D. lumholtzi to $100 \mathrm{pM}$ $5 \alpha$-CPS resulted in a relative tail spine length that was neither different from that of animals exposed to the negative control nor from animals exposed to EFI (Figure 3A).

In case of TCDCA, only the highest tested concentration of $190 \mu \mathrm{M}$ resulted in relative helmet lengths of $D$. lumholtzi that

TABLE 1 | Effect of extract of fish incubation water (EFI) and HPLC-fractions thereof on morphological parameters of Daphnia lumholtzi.

\begin{tabular}{|c|c|c|c|c|c|c|}
\hline Treatment & $\begin{array}{c}\text { It Relative HL } \\
{[\%]} \\
\text { (mean } \pm \text { SD) }\end{array}$ & $\begin{array}{c}\text { Tukey's } \\
\text { HSD }\end{array}$ & $\begin{array}{c}\text { Relative TL } \\
{[\%]} \\
\text { (mean } \pm \text { SD) }\end{array}$ & $\begin{array}{c}\text { Tukey's } \\
\text { HSD }\end{array}$ & $\begin{array}{l}\text { s Body length } \\
\text { [mm] } \\
\text { (mean } \pm \text { SD) }\end{array}$ & $\begin{array}{c}\text { Tukey's } \\
\text { HSD }\end{array}$ \\
\hline Control & $35.81 \pm 6.03$ & $C D$ & $84.73 \pm 14.62$ & C & $1.38 \pm 0.12$ & A \\
\hline $\mathrm{EFI}$ & $52.09 \pm 5.81$ & $A$ & $99.85 \pm 4.68$ & A & $1.43 \pm 0.08$ & A \\
\hline Fraction 1 & $42.31 \pm 5.90$ & $\mathrm{BC}$ & $92.38 \pm 6.30$ & $\mathrm{ABC}$ & $1.37 \pm 0.06$ & A \\
\hline Fraction 2 & $40.28 \pm 4.04$ & $B C D$ & $90.82 \pm 1.91$ & $\mathrm{ABC}$ & $1.39 \pm 0.05$ & A \\
\hline Fraction 3 & $48.15 \pm 5.90$ & $\mathrm{AB}$ & $97.92 \pm 4.18$ & $A B$ & $1.33 \pm 0.08$ & A \\
\hline Fraction 4 & $37.02 \pm 5.11$ & $C D$ & $91.60 \pm 4.23$ & $\mathrm{ABC}$ & $1.42 \pm 0.08$ & A \\
\hline Fraction 5 & $34.93 \pm 5.49$ & $C D$ & $84.69 \pm 10.15$ & C & $1.31 \pm 0.10$ & A \\
\hline Fraction 6 & $33.05 \pm 5.75$ & $\mathrm{D}$ & $88.91 \pm 5.93$ & $\mathrm{BC}$ & $1.40 \pm 0.07$ & A \\
\hline $\begin{array}{l}\text { One-way } \\
\text { ANOVA }\end{array}$ & $\begin{array}{c}F_{(7,65)}=14.05 \\
p<0.001\end{array}$ & & $\begin{array}{c}F_{(7,65)}=5.15 \\
p<0.001\end{array}$ & & $\begin{array}{c}F_{(7,65)}=2.26 \\
p=0.04\end{array}$ & \\
\hline
\end{tabular}

Relative length (length/body length ${ }^{\star} 100$ ) of helmet $(H L)$, tail spine $(T L)$ and absolute body length of Daphnia lumholtzi exposed to methanol as control, to EFI and HPLC fractions thereof, are depicted. Mean $\pm S D$ derive from animals at the time of their first reproduction, $n=8-10$. Significant differences of each morphological parameter between treatments after one-way ANOVA and Tukey's HSD test are indicated by different letters. Colored parameters are statistically different from animals exposed to the negative control and statistically equal to EFI exposed animals.

TABLE 2 | Concentration of 5 $\alpha$-cyprinol sulfate and taurochenodeoxycholic acid in bioassays in which either bile extract or two different concentrations of extract of fish incubation water (EFI) were used (Hahn et al., 2019).

\begin{tabular}{lccc}
\hline Compound & $\mathbf{1} \times$ EFI [nM] & $\begin{array}{c}\mathbf{1 : 5} \text { diluted EFI } \\
\text { [pM] }\end{array}$ & $\begin{array}{c}\text { Bile extract Cyprinus } \\
\text { carpio [nM] }\end{array}$ \\
\hline $\begin{array}{l}5 \alpha \text {-cyprinol sulfate } \\
\text { taurochenodeoxycholic }\end{array}$ & $0.14 \pm 0.01 \pm 0.08$ & $206 \pm 16$ & $1.13 \pm 0.02$ \\
acid (TCDCA) & & $28 \pm 2$ & \\
\hline
\end{tabular}

Bile extract originated from carp (Cyprinus carpio) and EFI from roach (Rutilus rutilus). Concentrations are depicted as mean $\pm S D$. were not distinguishable from those of animals exposed to EFI (Figure 3B). This threshold concentration of $190 \mu \mathrm{M}$ exceeded the concentration of TCDCA present in EFI $(140 \pm 10 \mathrm{pM})$ by more than six orders of magnitude. The tested range of concentrations of TCDCA from 19 pM to $190 \mu \mathrm{M}$ did not result in elongation of the relative tail spine length of $D$. lumholtzi (Figure 3B). In conclusion, the concentrations of TCDCA in fish incubation water (and EFI) were too low to affect helmet or spine length in D. lumholtzi.

\section{DISCUSSION}

Although the first kairomones responsible for the induction of defenses in two Daphnia species have been identified (Weiss et al., 2018; Hahn et al., 2019), it is not clear, how universal the perception of these compounds is among different Daphnia species and if these kairomones are used by invasive species as well. Besides the induction of morphological changes in D. lumholtzi it has already been demonstrated that $5 \alpha$-CPS induces DVM, a predator avoidance behavior, in D. magna (Hahn et al., 2019). The additional identification of $5 \alpha$-CPS as the fish-borne kairomone, that induces the formation of defensive morphological structures in D. lumholtzi, allows for speculation about universality of this kairomone, since it evidentially induces different defenses in different Daphnia species.

Our results in terms of the induction of morphological defenses in D. lumholtzi are in accordance with those concerning DVM induction in D. magna. This holds true not only for the finding that $5 \alpha$-CPS is the major kairomone but as well for the relevance of other bile salts (Hahn et al., 2019). Both defenses, DVM in D. magna and morphological defenses in D. lumholtzi, can also be induced by the bile salt TCDCA but in both cases the induction requires TCDCA concentrations that are five to six orders of magnitude higher than those found in fish incubation water (Hahn et al., 2019). Thus, one can hypothesize that structural similarity of TCDCA and $5 \alpha$-CPS may lead to nonspecific receptor binding of TCDCA to a $5 \alpha$-CPS receptor and that this receptor may be similar in D. magna and D. lumholtzi. Taken together, these findings suggest evolutionary conservancy in terms of the perception of fish-derived kairomones within the genus Daphnia. Furthermore, universality of the fish derived kairomone $5 \alpha$-CPS is suggested with respect to its ability to induce different defenses. So far $5 \alpha$-CPS has been shown to be the major fish-mediated kairomone with respect to the induction of DVM and morphological defenses. Still, the role of $5 \alpha$-CPS has not yet been tested with respect to the induction of life history changes (LHC). Pijanowska et al. (2020) showed for two bile salts that they induce LHCs in Daphnia albeit at fairly high concentrations of $1 \mathrm{mg} / \mathrm{L}$. It still remains to be demonstrated that fish exude the two investigated bile acids in biologically relevant concentrations, and since Pijanowska et al. (2020) did not include the bile alcohol $5 \alpha$-CPS in their study, a role of $5 \alpha$-CPS in induction of LHCs could not be ruled out yet.

Our results do not only support the hypothesis of universality of fish kairomones among Daphnia species, but also identified a chemical compound, which may be relevant for the invasion 


\section{A}

\section{5a-cyprinol sulfate [pM]}
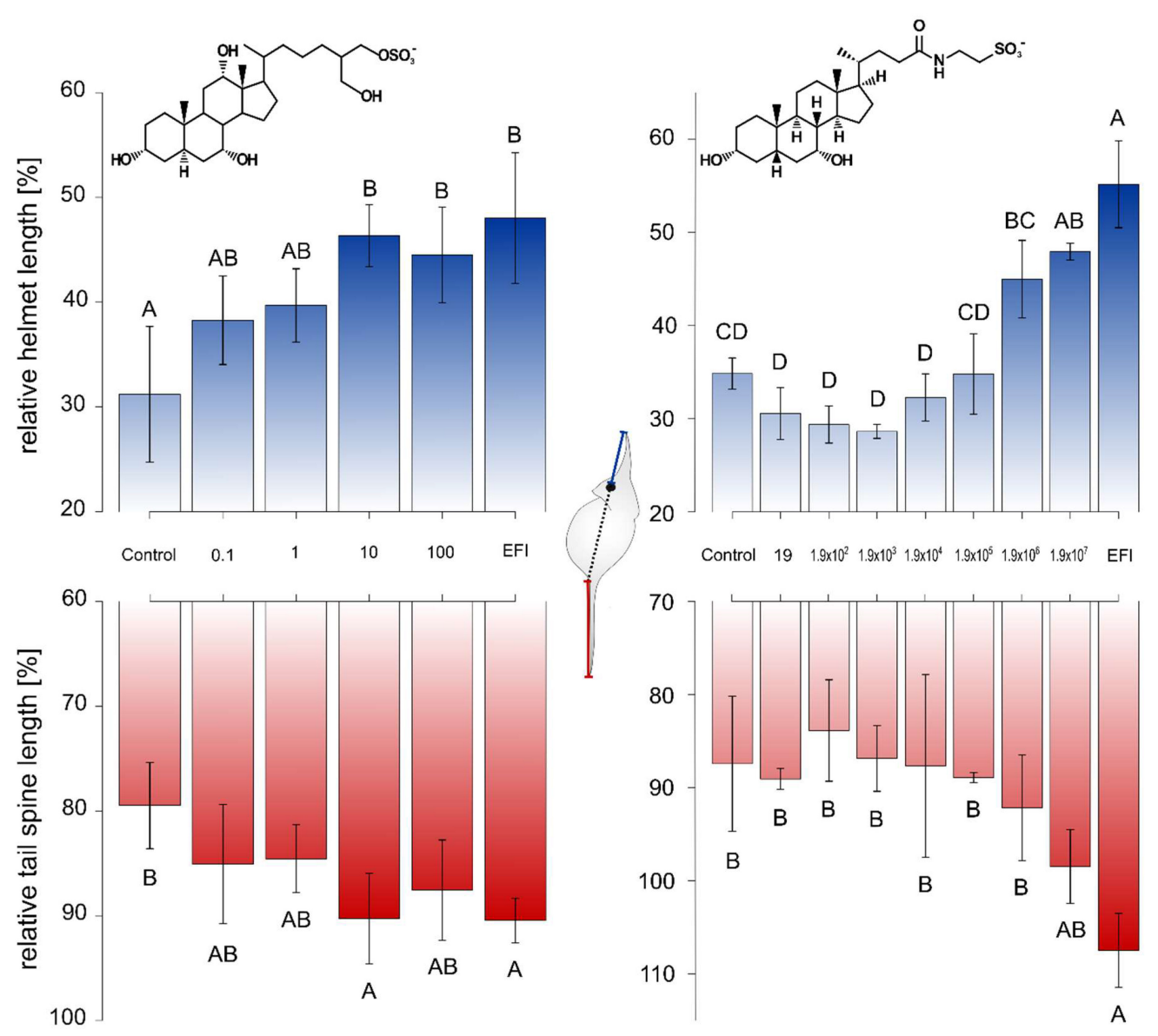

B

taurochenodeoxycholic acid [pM]

FIGURE 3 | Relative helmet and tail spine length of Daphnia lumholtzi in response to different bile salts. The relative length (length/body length*100) of helmet (blue) and tail spine (red) of $D$. lumholtzi exposed to methanol (Control), $\mathrm{C}_{18}$ solid-phase extract of incubation water of roach (R. rutilus; EFI), and concentration ranges of (A) $5 \alpha$-cyprinol sulfate and (B) taurochenodeoxycholic acid, is depicted. The scheme depicts the measurement of absolute helmet (blue) and tail spine (red) lengths. Mean \pm SD derive from animals at time of their first reproduction. Different letters indicate significant differences after one-way ANOVA and Tukey's HSD test $(\alpha<0.05)$ between treatments. (A) One-way ANOVA on relative helmet length: $F_{(5,2) 0}=6.98, p<0.001$, on relative tail spine length: $F_{(5,21)}=4.3, p<0.01$, $n=4-5$; (B) one-way ANOVA on relative helmet length: $F_{(8,18)}=30.7, p<0.001$, on relative tail spine length: $F_{(8,18)}=0.8, p>0.05, n=3$.

success of a Daphnia species. This reasoning is based on (Engel and Tollrian, 2012), who claimed that the induction of helmet and tail spine elongation in D. lumholtzi may facilitate its invasion success. More precisely, the elongated helmets lower the risk of being preyed upon by fish, as indicated by gut analyses of fish (Green, 1967) and by competition experiments of D. lumholtzi and D. pulicaria in presence of a planktivorous fish (Engel and Tollrian, 2009). Here elongation of the relative helmet length in $D$. lumholtzi was induced by extract of fish incubation water (EFI; R. rutilus, Cyprinidae), fish bile extract (Cyprinus carpio, Cyprinidae) and purified $5 \alpha$-CPS in a dose-dependent manner, but there are no similarly clear effects on the relative tail spine length in D. lumholtzi, which is in accordance with reports about tail spine elongation in response to the presence of fish (Tollrian, 1994; Dzialowski et al., 2003; Engel and Tollrian, 2009; Horstmann et al., 2021).
$5 \alpha$-CPS is the major bile salt in Cypriniformes (Hagey et al., 2010), which represent a very wide-spread order of fishes in fresh and even brackish water (Hastings et al., 2015). This bile alcohol is evolutionarily old, and from the perspective of Daphnia spp. $5 \alpha$-CPS is ideally suited as a kairomone as it is not specific for a single fish species, but instead its widespread release makes $5 \alpha$-CPS a perfect general chemical cue to estimate the risk of predation by fish (Von Elert and Loose, 1996; Von Elert and Pohnert, 2000). Our finding that $5 \alpha$-CPS is the kairomone that induces a phenotypic change in D. lumholtzi (spine elongation) and that $5 \alpha-C P S$ is responsible for the induction of DVM in Daphnia (Hahn et al., 2019) suggests that the underlying molecular mechanism of inducible defenses in Daphnia sp. is evolutionary conserved. Although $5 \alpha$-CPS efficiently induces morphological defenses in D. lumholtzi at 
picomolar concentrations, it is not yet confirmed that $5 \alpha$-CPS is the only fish-derived compound, which does so. The bile salt pattern is highly conserved between fish species of the same order, but differs among orders (Hagey et al., 2010). In line with this, the bile salt pattern of Cypriniformes is mainly dominated by $\mathrm{C}_{27}$ bile alcohols, especially by $5 \alpha$-CPS (Hagey et al., 2010). The only two studies reporting induction of (relative) helmet and tail spine length in D. lumholtzi have applied chemical cues from Cypriniformes (Tollrian, 1994; Engel and Tollrian, 2009), and thus most probably 5 $\alpha$-CPS (Hagey et al., 2010). Upon exposure to incubation water of Gasterosteus aculeatus (Perciformes) elongation of the helmet of D. lumholtzi was demonstrated (Graeve et al., 2021; Horstmann et al., 2021). Since bile salt patterns of Perciformes as for instance of $G$. aculeatus are characterized by the absence of $5 \alpha$-CPS (Hagey et al., 2010), it is well conceivable that in Perciformes bile salts other than $5 \alpha$-CPS induce morphological defenses in D. lumholtzi.

The fact that fish orders may differ with respect to whole groups of bile salts (Hagey et al., 2010), raises the question, if fish orders that lack $5 \alpha$-CPS do not induce the defensive morphological changes in D. lumholtzi. Gophen (1979) postulated that in Lake Kinneret (Israel), which had previously been dominated by Cypriniformes (Mirogrex terraesanctae), $D$. lumholtzi probably became extinct due to stocking the lake with Mugiliformes (Liza aurata, L. ramada) and Cichliformes (Sarotherodon aureus). Species of the order of Mugiliformes are only known to synthesize the $\mathrm{C}_{24}$ bile acids TCA and TCDCA and to lack $\mathrm{C}_{27}$ bile alcohols (Hagey et al., 2010), while the bile salt pattern of Cichliformes has not been elucidated yet. It can be speculated that stocking of the lake resulted in dominance of fish species perhaps lacking the spine elongating kairomone $\left(\mathrm{C}_{27}\right.$ alcohol), so that the degree of induced morphological defenses in D. lumholtzi then was too low to ensure co-existence with planktivorous fish, which led to the extinction of D. lumholtzi. This hypothesis is based on several assumptions, but considering the extensive invasion of D. lumholtzi (Havel et al., 1995), it is still worth investigation in future studies.

\section{CONCLUSION}

In conclusion, our study has revealed that $5 \alpha$-CPS, a single chemical released by fish, induces pronounced phenotypic changes in D. lumholtzi. Thus, the combination of this finding with previous ones demonstrates the multiplicity of $5 \alpha$-CPS as a cue in two ways: (i) $5 \alpha$-CPS is perceived by different Daphnia species and (ii) $5 \alpha$-CPS induces different defenses. This leads to the question, if $5 \alpha$-CPS is underlying further defenses of other Daphnia species as well. Apart from this, the identification of

\section{REFERENCES}

Bedrossiantz, J., Fuertes, I., Raldua, D., and Barata, C. (2021). Pharmacological modulation of fish-induced depth selection in D. magna: the role of cholinergic and GABAergic signalling. Sci. Rep. 11:19407. doi: 10.1038/s41598-021-988 $86-\mathrm{w}$ the kairomone used by the invasive species D. lumholtzi is of particular ecological interest, since its invasion success has earlier been indicated to be linked to the phenotypic plasticity, induced by the kairomone (Engel and Tollrian, 2009, 2012; Engel et al., 2014). In this context, it is of particular interest that $5 \alpha$-CPS is absent in the bile of various orders of fish (Hagey et al., 2010), raising the possibility that these fish, though preying on D. lumholtzi, may not induce morphological defenses and might thus provide a means of slowing down invasion by $D$. lumholtzi. As stated in a recent review, the field of inducible defenses in freshwater zooplankton is extremely complex and requires further multifaceted experiments to fill significant research gaps. Knowledge about kairomones is considered to be helpful in order to perform standardized experiments or to quantify kairomone concentrations in the field (Diel et al., 2020).

\section{DATA AVAILABILITY STATEMENT}

The raw data supporting the conclusions of this article are available upon request from the authors.

\section{AUTHOR CONTRIBUTIONS}

$\mathrm{MH}$ and EE conceived the study and wrote the manuscript. $\mathrm{MH}$ performed the experiments and analyzed the samples and the data. Both authors read and approved of the final version for submission.

\section{FUNDING}

This work was supported by a grant (EL 179/16-1) by the Deutsche Forschungsgemeinschaft (DFG) to EE.

\section{ACKNOWLEDGMENTS}

We would like to thank Geraldine Bennertz, Nils Heck and Christian Voss for their assistance with regard to animal cultivation and bioassay conduction.

\section{SUPPLEMENTARY MATERIAL}

The Supplementary Material for this article can be found online at: https://www.frontiersin.org/articles/10.3389/fevo.2022. 804521/full\#supplementary-material

Beyer, J. E., and Hambright, K. D. (2019). The niche and morphology of the invasive Daphnia lumholtzi in a subtropical reservoir, 20 years after invasion. J. Plank. Res. 41, 90-100. doi: 10.1093/plankt/fby053

Boersma, M., Spaak, P., and de Meester, L. (1998). Predator-mediated plasticity in morphology, life history, and behavior of Daphnia: the uncoupling of responses. Am. Nat. 152, 237-248. doi: 10.1086/286164 
Brett, M. T. (1992). Chaoborus and fish-mediated influences on Daphnia longispina population structure, dynamics and life history strategies. Oecologia 89, 69-77. doi: 10.1007/BF00319017

Diel, P., Kiene, M., Martin-Creuzburg, D., and Laforsch, C. (2020). Knowing the enemy: inducible defences in freshwater zooplankton. Diversity 12, 147. doi: $10.3390 / \mathrm{d} 12040147$

Dodson, S. (1988). The ecological role of chemical stimuli for the zooplankton: predator-avoidance behavior in Daphnia. Limnol. Oceanograp. 33, 1431-1439. doi: 10.4319/lo.1988.33.6part2.1431

Dzialowski, A. R., Lennon, J. T., O’Brien, W. J., and Smith, V. H. (2003). Predatorinduced phenotypic plasticity in the exotic cladoceran Daphnia lumholtzi. Fresh. Biol. 48, 1593-1602. doi: 10.1046/j.1365-2427.2003.01111.x

Effertz, C., and Von Elert, E. (2014). Light intensity controls anti-predator defences in Daphnia: the suppression of life-history changes. Proc. Royal Soc. B 281:20133250. doi: 10.1098/rspb.2013.3250

Engel, K., Schreder, T., and Tollrian, R. (2014). Morphological defences of invasive Daphnia lumholtzi protect against vertebrate and invertebrate predators. J. Plank. Res. 36, 1140-1145. doi: 10.1093/plankt/fbu023

Engel, K., and Tollrian, R. (2009). Inducible defences as key adaptations for the successful invasion of Daphnia lumholtzi in North America? Proc. Biol. Sci. 276, 1865-1873. doi: 10.1098/rspb.2008.1861

Engel, K., and Tollrian, R. (2012). Competitive ability, thermal tolerance and invasion success in exotic Daphnia lumholtzi. J. Plank. Res. 34, 92-97. doi: 10.1093/plankt/fbr083

Gophen, M. (1979). Extinction of Daphnia lumholtzi (SARS) in Lake Kinneret (Israel). Aquaculture 16, 67-71. doi: 10.1016/0044-8486(79)90173-x

Graeve, A., Janßen, M., Villalba De La Pena, M., Tollrian, R., and Weiss, L. C. (2021). Higher, faster, better: maternal effects shorten time lags and increase morphological defenses in Daphnia lumholtzi offspring generations. Front. Ecol. Evol. 9:637421. doi: 10.3389/fevo.2021.637421

Green, J. (1967). The distribution and variation of Daphnia lumholtzi (Crustacea: Cladocera) in relation to fish predation in Lake Albert. East Afr. J. Zoo. 151, 181-197. doi: 10.1111/j.1469-7998.1967.tb02872.x

Hagey, L. R., Møller, P. R., Hofmann, A. F., and Krasowski, M. D. (2010). Diversity of bile salts in fish and amphibians: evolution of a complex biochemical pathway. Physiol. Biochem. Zool. 83, 308-321. doi: 10.1086/649966

Hahn, M., Von Elert, E., Bigler, L., Hernández, D., and Schloerer, N. E. (2018). 5 $\alpha$-Cyprinol sulfate: complete NMR assignment and revision of earlier published data, including the submission of a computer-readable assignment in NMReDATA format. Mag. Reson. Chem. 56, 1201-1207. doi: 10.1002/mrc.4782

Hahn, M. A., Effertz, C., Bigler, L., and Von Elert, E. (2019). 5a-cyprinol sulfate, a bile salt from fish, induces diel vertical migration in Daphnia. eLife 8:e44791. doi: 10.7554/eLife.44791.001

Hahn, M. A., and von Elert, E. (2020). The impact of diel vertical migration on fatty acid patterns and allocation in Daphnia magna. Peerj 8:e8809. doi: 10.7717/peerj.8809

Hastings, P. A., Walker, H. J., and Galland, G. R. (2015). Fishes: A guide to their diversity. California: Univ of California Press.

Havel, J. E., Mabee, W. R., and Jones, J. R. (1995). Invasion of the exotic cladoceran Daphnia lumholtzi into North American reservoirs. Can. J. Fish. Aqu. Sci. 52, 151-160. doi: 10.1139/f95-015

Horstmann, M., Tollrian, R., and Weiss, L. C. (2021). Thwarting predators? A three-dimensional perspective of morphological alterations in the freshwater crustacean Daphnia. PLoS One 16:e0254263. doi: 10.1371/journal.pone.0254263

Macháček, J. (1991). Indirect effect of planktivorous fish on the growth and reproduction of Daphnia galeata. Hydrobiologia 225, 193-197.
Pijanowska, J., Markowska, M., Ruszczyńska, A., Bulska, E., Dawidowicz, P., Ślusarczyk, M., et al. (2020). Kairomone-like activity of bile and bile components: a step towards revealing the chemical nature of fish kairomone. Sci. Rep. 10:7037. doi: 10.1038/s41598-020-63456-z

R Core Team (2019). R: A Language and Environment for Statistical Computing. Vienna: R Foundation for Statistical Computing.

Sorensen, K. H., and Sterner, R. W. (1992). Extreme cyclomorphosis in Daphnia lumholtzi. Freshwater Biol. 28, 257-262.

Tollrian, R. (1994). Fish-kairomone induced morphological changes in Daphnia lumholtzi (Sars). Archiv. Für. Hydrobiol. 130, 69-75. doi: 10.1127/archivhydrobiol/130/1994/69

Von Elert, E., and Jüttner, F. (1997). Phosphorus limitation and not light controls the extracellular release of allelopathic compounds by Trichormus doliolum (Cyanobacteria). Limnol. Oceanograp. 42, 1796-1802. doi: 10.4319/lo.1997.42. 8.1796

Von Elert, E., and Loose, C. J. (1996). Predator-induced diel vertical migration in Daphnia: enrichment and preliminary chemical characterization of a kairomone exuded by fish. J. Chem. Ecol. 22, 885-895. doi: 10.1007/BF0202 9942

Von Elert, E., and Pohnert, G. (2000). Predator specificity of kairomones in diel vertical migration of Daphnia: a chemical approach. Oikos 88, 119-128. doi: 10.1034/j.1600-0706.2000.880114.x

Von Elert, E., and Stibor, H. (2006). Predator-mediated life history shifts in Daphnia: enrichment and preliminary chemical characterisation of a kairomone exuded by fish. Archiv. Für. Hydrobiol. 167, 21-35.

Wacker, A., and Elert, V. (2001). Polyunsaturated fatty acids: evidence for nonsubstitutable biochemical resources in Daphnia galeata. Ecology 82, 2507-2520. doi: $10.2307 / 2679932$

Weiss, L. C., Albada, B., Becker, S. M., Meckelmann, S. W., Klein, J., Meyer, M., et al. (2018). Identification of Chaoborus kairomone chemicals that induce defences in Daphnia. Nat. Chem. Biol. 14, 1133-1139. doi: 10.1038/s41589-0180164-7

Weiss, L. C., Kruppert, S., Laforsch, C., and Tollrian, R. (2012). Chaoborus and Gasterosteus anti-predator responses in Daphnia pulex are mediated by independent cholinergic and gabaergic neuronal signals. PLoS One 7:e36879. doi: 10.1371/journal.pone.0036879

Whittington, D., and Walsh, M. R. (2015). Divergent phenotypic responses to predators and cyanobacteria in Daphnia lumholtzi. Freshwater Biol. 60, 18801889. doi: $10.1111 /$ fwb. 12618

Conflict of Interest: The authors declare that the research was conducted in the absence of any commercial or financial relationships that could be construed as a potential conflict of interest.

Publisher's Note: All claims expressed in this article are solely those of the authors and do not necessarily represent those of their affiliated organizations, or those of the publisher, the editors and the reviewers. Any product that may be evaluated in this article, or claim that may be made by its manufacturer, is not guaranteed or endorsed by the publisher.

Copyright (c) 2022 Hahn and von Elert. This is an open-access article distributed under the terms of the Creative Commons Attribution License (CC BY). The use, distribution or reproduction in other forums is permitted, provided the original author(s) and the copyright owner(s) are credited and that the original publication in this journal is cited, in accordance with accepted academic practice. No use, distribution or reproduction is permitted which does not comply with these terms. 